\title{
A review on applications of heuristic optimization algorithms for optimal power flow in modern power systems
}

\author{
Ming NIU, Can WAN, Zhao XU ( $\bowtie)$
}

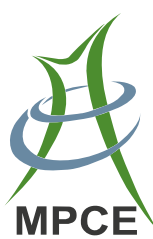

\begin{abstract}
Optimal power flow (OPF) is one of the key tools for optimal operation and planning of modern power systems. Due to the high complexity with continuous and discrete control variables, modern heuristic optimization algorithms (HOAs) have been widely employed for the solution of OPF. This paper provides an overview of the latest applications of advanced HOAs in OPF problems. The most frequently applied HOAs for solving the OPF problem in recent years are covered and briefly introduced, including genetic algorithm (GA), differential evolution (DE), particle swarm optimization (PSO), and evolutionary programming (EP), etc.
\end{abstract}

Keywords Heuristic optimization algorithm, Optimal power flow, Multi-objective optimization, Constraint optimization

\section{Introduction}

For the stable and economic operation of power systems, optimal power flow (OPF) aims to find the optimal settings of a given power system network that optimize a certain objective function while satisfying its power flow equations, system security, and equipment operating limits [1].

CrossCheck date: 27 November 2014

Received: 16 September 2014/Accepted: 1 December 2014/

Published online: 11 December 2014

(C) The Author(s) 2014. This article is published with open access at Springerlink.com

M. NIU, C. WAN, Z. XU, The Hong Kong Polytechnic

University, Hong Kong, China

$(\bowtie)$ e-mail: zhao.xu@polyu.edu.hk

M. NIU

e-mail: zimo.niu@connect.polyu.hk

C. WAN

e-mail: can.wan@connect.polyu.hk
The most commonly used objective is the minimization of the overall fuel cost function. In addition, there are several other objectives are to minimize the active power loss, bus voltage deviation, emission of generating units, number of control actions, and load shedding [2]. In practical power system operation, the OPF problem adjusts the continuous control variables (e.g. real power outputs and voltages) and discrete control variables (e.g. transformer tap setting, phase shifters, and reactive injections) to reach the optimal objective function while satisfying a set of physical and operational constraints. Therefore, it is a highly constrained, mixed-integer, non-linear and non-convex optimization problem.

Conventional techniques such as linear programming (LP), quadratic programming (QP), and non-linear programming (NLP), were developed to solve the OPF problems with some theoretical assumptions, such as convexity, differentiability, and continuity, which may not be suitable for the actual OPF conditions. In addition, the convergence to the global or local optimal solution is highly dependent on the selected initial guess [3]. Moreover, continuous LP, QP, and NLP formulations cannot accurately model discrete control variables, such as transformer tap ratios or switched capacitor banks. Mixed integer linear programming (MILP) techniques were introduced to solve this problem [4]. However, the nonlinearity of the power system cannot be fully represented by MILP formulations, and therefore cause inherent inaccuracy. To overcome these drawbacks, HOAs such as genetic algorithm (GA), evolutionary programming (EP), simulated annealing (SA), tabu search (TS), particle swarm optimization (PSO), differential evolution (DE), etc., have been employed for the solution of the OPF problem. The results reported in previous literatures were promising and encouraging for further research in this area.

This paper provides an extensive coverage of the major research work that make use of HOAs to the OPF problem. 
The remainder of this paper is structured as follows. A brief introduction of the most frequently used HOAs (GA, EP, $\mathrm{PSO}, \mathrm{DE}$, etc) for the OPF problem is given in Section 2. The generalized formulation of the OPF problem is expressed in Section 3. Section 4 provides a detailed investigation to OPF related research work applying HOAs. Section 5 gives a discussion on the status and trend of HOA application on OPF problem. Finally, this paper is summarized in Section 6.

\section{Modern heuristic optimization algorithms}

The modern HOAs represent a group of intelligent algorithms that either make analog of the natural evolution process based on Darwinian principles or mimic a certain natural phenomenon in searching for an optimal solution. They have been successfully applied to a wide range of power system optimization problems where non-differentiable regions exist and the global solution are extremely difficult to be gauged. The most popularly used HOAs in solving OPF problem are compactly introduced as the follows.

1) Genetic algorithm: GA is one of the most popular and famous approaches in evolutionary computation. Founded on the mechanism of natural genetics and Darwinian principles of evolution and natural selection, this novel algorithm showed strong capabilities and advantages for solving a wide range of problems as introduced in [5]. GA can be considered as a population-based approach, the search process of which is conducted by means of transforming a set of points (individuals) to another set of points in the search space. In the original GA, each individual is represented via a fixed-length binary string. This method maps the points in the search space into the instances of artificial chromosome. Desired precision can be simply approximated through tuning the length of binary string. The strong preference to the binary representation of GA probably derives from Schema Theorem [6] which tries to investigate the mathematical foundation of GA.

2) Particle swarm optimization: PSO, which was introduced in $[7,8]$ in 1995 , is one of the most important swarm intelligence paradigms. The PSO uses a simple mechanism that mimics swarm behavior in birds flocking and fish schooling to guide the particles to search for globally optimal solution. As PSO is easy to implement, it has rapidly progressed in recent years and with many successful applications in solving realworld optimization problems.

3) Differential evolution: The DE approach is firstly proposed in a technical report in 1995 [9]. It is a population-based method and is generally considered a parallel stochastic direct search optimizer that is simple yet powerful. DE is a stochastic populationbased optimization algorithm with real parameters and real-valued functions. The core idea behind DE is a scheme for generating trial parameter vectors. DE generates new parameter vectors by weighing the difference vector between two population members and then adding that to a third member. If the resulting vector yields a lower objective function value than a previously determined population member, the newly generated vector replaces the vector to which it was compared. In comparisons to most other HOAs, the DE algorithm is much simpler and more straightforward to implement. The main body of the algorithm takes four or five lines of code in any programming language. Despite its simplicity, the gross performance of DE in terms of accuracy, convergence rate and robustness makes it attractive for applications to various real-world optimization problems [10-12], where finding an approximate solution in a reasonable amount of computational time is of considerable importance. The spatial complexity of DE is lower than that of some highly competitive real parameter optimizers. This feature helps in extending DE to handle expensive and large-scale optimization problems.

4) Evolutionary programming: EP was first introduced in the research of artificial intelligence [13]. In order to achieve intelligent behavior, there came an idea of defining the environment as a sequence of symbols (in a finite alphabet) and evolving an algorithm to predict the next symbol to appear based on the former observed sequence of symbols. Finite state machine (FSM) is chosen to be the form of individuals, as it provides a meaningful representation for the required behaviors in the environment. While the original form of EP was applied in discrete problems due to the FSM representation, EP was extended into the real-valued continuous optimization problem [14]. Both the mutation mode and the number of mutations per offspring FSM are with respect to a probability distribution, which means some individual may mutates more than once in one generation.

In addition to the abovementioned HOAs, ant colony optimization (ACO) [15], artificial neural network (ANN) [16], simulated annealing (SA) [17], tabu search (TS) [18], quantum-inspired evolutionary algorithm (QEA) [19, 20], and artificial bee colony (ABC) [21] are also popular HOA applications with regard to the OPF problem. Thus, corresponding literature reviews are also included in Section 4. 


\section{Problem formulation}

This section provides a generalized OPF problem description. It should be noted that OPF formulations differ greatly depending on the different selection of variables, objectives and constraints. Due to this aspect of OPF problem, the formulation selected often has impacts for both algorithm design and solution accuracy.

From the perspective of mathematics, the OPF optimize a constructed objective subject to different sets of equality and inequality constraints. Without loss of generality, the problem can be formulated as follows:

$\operatorname{Min} f(\mathrm{x}, u)$

Subject to

$g(x, u)=0$

$h(x, u) \leq 0$

where $f()$ is the objective function to be minimized; $x$ denotes the dependent or state variables including active power output of the slack bus $P_{G_{1}}$, voltage of the load buses $V_{L}$ (magnitudes and phase angles), generator reactive power outputs $Q_{\mathrm{G}}$, and transmission line flow $S_{l}$, expressed as

$x^{\mathrm{T}}=\left[P_{G_{1}}, V_{L_{1}} \cdots V_{L_{N L}}, Q_{G_{1}} \cdots Q_{G_{N G}}, S_{l_{1}} \cdots S_{l_{n l}}\right]$

where $N L, N G$ and $n l$ denote the number of load buses, generators and transmission lines, respectively; $u$ represents the independent variables, also known as control variables consisting of voltages at generation buses $V_{G}$ (magnitudes), active power generation $P_{G}$ at PV buses, transformer tap settings $T$, and reactive power injections $Q_{C}$ by the shunt volt-amperes reactive (VAR) compensations, represented as

$u^{\mathrm{T}}=\left[P_{G_{2}} \cdots P_{G_{N G}}, V_{G_{1}} \cdots V_{G_{N G}}, Q_{C_{1}} \cdots Q_{C_{N C}}, T_{1} \cdots T_{N T}\right]$

where $N C$ and $N T$ are the number of the VAR compensators and regulating transformers, respectively. The control variables can be divided into two categories, discrete $u_{d}$ and continuous $u_{c}$, given by

$u_{d}^{\mathrm{T}}=\left[T_{1} \cdots T_{N}, Q_{C_{1}} \cdots Q_{C_{N}}\right]$

$u_{c}^{\mathrm{T}}=\left[P_{G_{2}} \cdots P_{G_{N}}, V_{G_{2}} \cdots V_{G_{N}}\right]$

In practice, the OPF problem has two types of constraints, i.e., the equality and inequality constraints. The equality constraints, defined by (2), are a set of nonlinear power flow equations, represented as

$P_{G_{i}}-P_{D_{i}}-P_{B_{i}}(V)=0$

$Q_{G_{i}}-Q_{D_{i}}-Q_{B_{i}}(V)=0$

where $P_{G_{i}}$ and $Q_{G_{i}}$ are active and reactive power generated at bus $i$, respectively; $P_{D_{i}}$ and $Q_{D_{i}}$ represent the load demand at the same bus; and $P_{B_{i}}$ and $Q_{B_{i}}$ denote the total sending and receiving power at each bus.

The inequality constraints, represented in (3), are a set of continuous and discrete constraints that define the system operational and security limits, including:

1) Generation constraints:

To ensure stable operation, generation bus voltages, active power and reactive power outputs are restricted by their lower and upper limits as

$$
\begin{aligned}
& V_{G_{i}}^{\min } \leq V_{G_{i}} \leq V_{G_{i}}^{\max }, \quad i=1, \cdots, N G \\
& P_{G_{i}}^{\min } \leq P_{G_{i}} \leq P_{G_{i}}^{\max }, \quad i=1, \cdots, N G \\
& Q_{G_{i}}^{\min } \leq Q_{G_{i}} \leq Q_{G_{i}}^{\max }, \quad i=1, \cdots, N G
\end{aligned}
$$

2) Transformer constraints:

The discrete transformer tap settings are restricted by their limits as

$T_{i}^{\min } \leq T_{i} \leq T_{i}^{\max }, \quad i=1, \cdots, T_{N}$

3) Shunt VAR constraints:

The discrete reactive power injections due to capacitor banks are restricted by their lower and upper limits defined as

$Q_{C_{i}}^{\min } \leq Q_{C_{i}} \leq Q_{C_{i}}^{\max }, \quad i=1, \cdots, C_{N}$

4) Security constraints:

The constraints of voltages at load buses as well as the transmission lines loading should be ensured as

$$
\begin{aligned}
& V_{L_{i}}^{\min } \leq V_{L_{i}} \leq V_{L_{i}}^{\max }, \quad i=1, \cdots, N L \\
& S_{l_{i}} \leq S_{l_{i}}^{\max }, \quad i=1, \cdots, n l
\end{aligned}
$$

\section{Modern heuristic algorithms for the OPF}

This section provides a detailed survey on the OPF related research works with the applications of GA, PSO, DE, EP, and some other commonly used HOAs.

\subsection{Genetic algorithm based approach}

GA was successfully used for optimal reactive power planning in [22] to search for a global optimal solution. It has been verified on practical 51-bus and 224-bus systems to indicate its feasibility and capability. An improved genetic algorithm (IGA) with the dynamical hierarchy of the coding system was developed to solve the OPF problem [23]. The IGA demonstrate the ability to code a large number of control variables in a practical system. It was tested on the IEEE 30-bus system with both normal and contingent operation states. OPF problem for a multi-node auction market was studied by means of GA in [24] to maximize the total participants' benefit at all nodes in the power system. In [25], a self-adaptive real coded genetic 
algorithm (SARGA) was developed to solve OPF problem, where the self-adaptation in real coded genetic algorithm was reached through simulated binary crossover operator. A novel evolutionary algorithm was developed combing a new decoupled quadratic load flow (DQLF) solution with enhanced genetic algorithm (EGA) to solve the multiobjective OPF problem [26]. A strength pareto evolutionary algorithm (SPEA) based approach was employed to obtain the Pareto-optimal set. The proposed multi-objective evolutionary algorithm demonstrates superiority in comparisons to PSO-Fuzzy approach. An adaptive genetic algorithm (AGA) was developed to solve OPF problems and voltage control [27], where the probabilities of crossover and mutation were adjusted in terms of the fitness values of the solutions and the normalized fitness distances between the solutions in the evolution process. In [28], a refined genetic algorithm (RGA) was developed for solving OPF problem. This GA can code a large number of control variables and has less sensitivity to starting points. GA was also used to deal with power system security enhancement based OPF in [29] considering the actions to possible overloads in the network due to contingencies. An enhanced genetic algorithm (EGA) with advanced and problem-specific operators was introduced for solving OPF with both continuous and discrete control variables [30]. An efficient real-coded mixed-integer genetic algorithm (MIGA) was presented in [31] to solve non-convex OPF problems with security constraints. According to the numerical studies on 26-bus and the IEEE 57-bus systems, the MIGA performs better than the EP. A novel hybrid method integrating a GA with a nonlinear interior point method (IPM) was proposed for OPF problem [32]. In the hybrid approach, GA was responsible for solving the discrete optimization with the continuous variables, and the IPM is responsible for solving the continuous optimization with the discrete variables. Numerical simulations were implemented on IEEE 30-bus, IEEE 118-bus and realistic Chongqing 161-bus test systems. Reference [33] discussed the effects of various combination of control variables on the convergence of simple genetic algorithm. Statistical parameter based study was conducted to visualize the effects of the selection of control variables on OPF convergence in terms of the computation time and the accuracy improvement. The experiment results proved that the set of control variables with the voltage of slack bus, the active/reactive power outputs of generators, and the reactive power outputs of controllable buses can be the most effective in obtaining the global solution under normal and contingent conditions. In [34], it was claimed that the main disadvantages of GAs was the high CPU execution time and the qualities of the solution deteriorate with practical large-scale OPF problems. An efficient parallel GA was developed for the solution of large-scale OPF problem with the consideration of practical generators constraints. The length of the original chromosome was reduced on basis of the decomposition level and adapted with the topology of the new partition. Partial decomposed active power demand was added as a new variable and searched within the active power generation variables of the new decomposed chromosome. The strategy of the OPF problem was decomposed into two sub-problems, of which the first subproblem was related to active power planning to minimize the fuel cost function and the second sub-problem was designed to make corrections to the voltage deviation and reactive power violation in an efficient reactive power planning of multi static var compensator (SVC). Numerical results on three test systems IEEE 30-bus, IEEE 118-bus and 15 generation units with prohibited zones were presented and compared with results of stochastic search algorithms, enhanced GA, ant colony optimization, and GA-fuzzy system approach.

\subsection{Particle swarm optimization based approach}

A novel particle swarm optimization approach based on multi-agent systems (MAPSO) was presented [35] to solve OPF problems. Each agent, representing a particle to PSO, in MAPSO competes and cooperates with its neighbors. Experiment results prove that the proposed MAPSO approach can reach better solutions much faster than the mature approaches. A multi-objective PSO technique was developed to deal with the highly nonlinear and non-convex multi-objective OPF problem [36]. In addition to the conventional objective generation cost, another conflicting objective environmental pollution is formulated and minimized simultaneously. A fuzzy based hybrid PSO approach for solving OPF problem considering the forecasting uncertainties of wind speed and load demand in power systems was proposed in [37]. A comprehensive learning PSO (CLPSO) was developed to reactive power dispatch to reduce grid congestions [38]. A new multi-objective PSO (MOPSO) technique for solving OPF problem was proposed in [39]. The proposed MOPSO methodology is formulated via the redefinition of global best and local best individuals in multi-objective optimization domain. Reference [40] presented a hybrid particle swarm optimization algorithm (HPSO) to solve the discrete OPF problem. Newton-Raphson algorithm for the minimization of the mismatch of the power flow equations was integrated to the proposed HPSO algorithm. PSO technique was applied for the transient-stability constrained OPF (TSCOPF) problem modeled as an extended OPF with additional rotor angle inequality constraints [41]. Reference [42] proposed a PSO algorithm with reconstruction operators to solve the $\mathrm{OPF}$ problem with embedded security constraints (OPF-SC), represented by a mixture of continuous and discrete control 
variables. The major objective is to minimize the total operating cost, taking into account both operating security constraints and system capacity requirements. The reconstruction operators guarantee searching the optimal solution within the feasible space, reducing the computation time and improving the quality of the solution. An improved PSO algorithm was developed for multi-objective OPF problem. The improved PSO that profits from chaos queues and self-adaptive concepts was used to improve the quality of the solution, particularly to avoid being trapped in local optima. In addition, a new mutation strategy combining different mutant rules was proposed to increase the search ability of the proposed algorithm. The proposed multi-objective OPF considers the fuel cost, loss, voltage stability and emission impacts involved in the objective functions. A fuzzy decision-based mechanism was used to select the best compromise solution of Pareto set obtained by the proposed PSO. In [43], PSO and group search optimizer (GSO) were used to solve the OPF problem with distributed generator failures in power networks. An OPF problem considering controllable and uncontrollable distributed generators was formulated, and cases with single and multiple generator failures were addressed.

\subsection{Differential evolution based approach}

A multi-agent based differential evolution (MADE) based on multi-agent systems was developed for dealing with OPF problem with non-smooth and non-convex generator fuel cost curves in [44]. A novel robust differential evolution algorithm (RDEA) with new recombination operator was introduced to solve multi-objective OPF problem including two objective functions of generation cost and voltage stability margin, for OPF problem [45]. Similarly, DE was used to solve OPF problem with multiple and competing objectives [46]. The OPF problem was divided into two sub-problems, i.e, active power dispatch and reactive power dispatch were considered. A DE-based approach to solve the OPF problem was developed in [47]. In their formulation, different objective functions that reflect fuel cost minimization, voltage profile improvement, and voltage stability enhancement were examined. Non-smooth pricewise quadratic cost function was also been considered. Reference [48] proposed a similar formulation of OPF with non-smooth and non-convex generator fuel cost curves. They employed a modified DE with a more exploitative mutation strategy and a random mutant factor. For testing purpose, the authors adopted a six-bus and the IEEE 30 bus test systems with three different types of generator cost curves. Comparisons were made among EP, PSO, typical DE, and results were in favor of the proposed modified DE. In [49], DE was comprehensively studied in terms of concept, mechanism, and parameter setting for solving OPF problems. The effectiveness of parallel computing technology for speeding up the computation was also analyzed. It has been concluded that DE requires relatively large populations to avoid premature convergence for medium-size test systems. In order to overcome this disadvantage, a decomposition and coordination method was proposed by the same authors based on the cooperative co-evolutionary architecture and the voltage-var sensitivity-based power system decomposition technique and incorporated with DE in [50]. The framework was implemented with a three-level parallel computing topology. Basu has used DE to minimize the generator fuel cost in OPF with flexible AC transmission systems (FACTS) devices including thyristor-controlled series capacitor (TCSC) and thyristor-controlled phase shifter (TCPS) [51]. Comparisons among DE, EP, and GA were conducted, indicating that the DE approach can obtain better solution and less computational complexities. Considering the transient stability constraints in OPF, In [52], DE was used to find the optimal setting for power system operation. To deal with the large-scale system and speed up the computation, DE was parallelized and implemented on a Beowulf PC-cluster. Reference [53] proposed a hybrid algorithm combining sequential quadratic programming (SQP) and DE for the solution of the OPF problem. In this hybrid method, SQP was used to generate an individual, which is a member of an initial population, for DE algorithm. This manipulation made DE more effectively to reach the optimal solution.

\subsection{Evolutionary programming}

In [54] and [55], an efficient and reliable EP algorithm was developed to solve OPF problem using the gradient information. The proposed algorithm has been successfully tested on IEEE 30-bus system with different highly nonlinear curves of generator performance. An improved EP and its hybrid version combined with the nonlinear interior point technique were proposed in [56] to solve the optimal reactive power dispatch problems, indicating the superiority of computational efficiency and optimality. The common practices in regulating reactive power were integrated in modifying the mutation direction of control variables of EP to improve its speed. The interior point method was applied to reach a fast initial solution which assisted the initial population of the improved EP method. An improved EP with multiple subpopulations and parallel search for solving OPF with non-smooth and non-convex generator fuel cost curves was proposed in [57]. Gaussian and Cauchy mutation operators have been included in different subpopulations to improve the search diversity and avoid the local optimum. In [58], EP was applied for 
solving security constrained optimal power flow (SCOPF) problem, where contingency-case security constraints were involved in the optimization of the defined objective function. The EP based OPF in deregulated electric market environment was used and validated in [59]. In [60], EP algorithm was proposed to solve the OPF problem of generator units with ramp rate limits and non-smooth fuel functions such as quadratic, piece-wise, valve point loading and combined cycle cogeneration plants.

\subsection{Other techniques}

In [61], artificial neural networks (ANNs) have been employed to model stability and security constraints in OPF to formulate the system security boundary (SB). The key novelties of the proposed algorithm include that a $\mathrm{NN}$ is trained to derive the SB model and a differentiable mapping function obtained from the $\mathrm{NN}$ is used as a constraint in the formulation of OPF problem. This approach ensures that the operating points resulting from the $\mathrm{OPF}$ solution process are within a feasible and secure region, comparing with typical security-constrained OPF models. A new ANN memory model-based algorithm was proposed to online implement for solving the unified OPF [62]. The ANN memory model was used to store the load patterns and their related optimal schedules. The proposed algorithm maximizes voltage stability margin while minimizing two other objectives generation cost and transmission loss.

Different ant colony optimization (ACO) algorithms were proposed to handle optimal reactive power dispatch problem in [63], including Ant system (AS), elitist ant system (EAS), rank-based ant system (ASrank) and maxmin ant system (MMAS). The problem was modeled as a combinatorial optimization problem involving nonlinear objective function with multiple local minima. The proposed ACO algorithms have been compared to conventional mathematical methods, i.e. genetic algorithm, evolutionary programming, and particle swarm optimization to demonstrate the effectiveness and efficiency. An ant colony system (ACS) method for constrained load flow problem was proposed in [64]. The proposed ACS is a distributed algorithm consisting of a set of cooperating ants to collaboratively search an optimum solution of the constrained load flow problem. In addition, the ACS algorithm was also applied to the reactive power control problem with network operating constraints to minimize real power losses.

Simulated annealing (SA) technique was proposed for solving OPF in [65]. It has been demonstrated that SA is able to solve the OPF problem as well as the load flow and the economic dispatch problem simultaneously. In [66], a novel HOA algorithm, called biogeography based optimization (BBO) was employed to solve constrained OPF problems in power systems with the consideration of valve point nonlinearities of generators. The simulation results of the proposed approach have been compared with EP, GA, PSO, mixed-integer particle swarm optimization (MIPSO) and sequential quadratic programming (SQP) to indicate its effectiveness for the global optimization of multi-constraint OPF problems. In [67], a quantum-inspired evolutionary algorithm (QEA) based on quantum computation was developed for bid-based active and reactive $\mathrm{OPF}$ problems. In [68], artificial bee colony (ABC) algorithm based on the intelligent foraging behavior of honeybee swarm was proposed for optimal reactive power flow (ORPF) problem.

\subsection{Hybrid approach}

A hybrid tabu search and simulated annealing (TS/SA) approach was proposed in [69] to deal with OPF control with FACTS devices including two types TCSC and TCPS. Test results on IEEE 30-bus system demonstrate that the proposed hybrid TS/SA approach can perform better than GA, SA, or TS alone. A hybrid approach integrating fuzzy systems with GA and PSO algorithm was proposed for the application for OPF problem [70]. A hybrid algorithm of DE and EP (DEEP) was proposed for solving ORPF problem [71]. The proposed DEEP algorithm reduces the required population size by using the advantages of $\mathrm{DE}$ and EP. In order to overcome the limits of DE and artificial bee colony (ABC), a hybrid $\mathrm{DE}$ and $\mathrm{ABC}$ technique (DE-ABC) was developed for solving the ORPF problem [72]. Numerical tests indicate the robustness of the DE-ABC approach. A hybrid evolving ant direction differential evolution (EADDE) algorithm was developed to deal with the OPF problem with non-smooth and non-convex generator fuel cost characteristics in [73].

\section{Discussion}

HOAs are typically very versatile with respect to OPF problem format. In addition, most HOAs are able to escape local optima which is critical for solving OPF problem.

However, all the HOAs discussed tend to be computationally intensive, yielding impractically long execution times for OPF problems involving large scale systems. To overcome this, parallel processing was executed by most of the reviewed population based HOAs, therefore, the computational time can be significantly reduced.

Furthermore, the reviewed HOAs possess several parameters which must be tuned to ensure good performance. Consideration of the tuning of pre-determined parameters of HOAs and the time limitation of online OPF operation will make these algorithms less robust. Hence, one of the most challenging aspects for HOAs lies in how 
to consistently converge to a feasible solution that provides an acceptable objective value within a limited function evaluations.

In the reviewed literatures, on the premise of a proper pre-defined parameter choices, almost every HOA method is claimed as being more robust or can converge to a better solution compared with other HOAs. However, comparisons between different HOAs are difficult, as the selection of pre-defined parameters for each HOAs dominates the results. Moreover, according to the no-free-lunch (NFL) theorem, there cannot exist any algorithm for solving all problems that is generally superior to its peers [74]. Therefore, the authors suggest that HOAs should be designed with respect to solving a specific aspect or formulation of OPF problem, i.e. MINLP formulation, so as the comparisons being made.

\section{Conclusions}

This paper presents relevant research work applying HOAs for solving the OPF problem. It highlights the difficulties of deterministic methods facing i.e. non-continuity and non-differentiability of the OPF objective function. The reviewed articles are organized according to different categories of HOAs. A brief discussion of the limitations and trends of HOA applications with respect to OPF problem is presented in addition to the corresponding literature reviews.

Acknowledgment This work was partially supported by Hong Kong RGC Theme Based Research Scheme Grants No. T23-407/ $13 \mathrm{~N}$ and T23-701/14 N. The work of Ming NIU was supported by a Ph.D. Research Studentship. The work of Can WAN was supported by a Hong Kong Ph.D. Fellowship.

Open Access This article is distributed under the terms of the Creative Commons Attribution License which permits any use, distribution, and reproduction in any medium, provided the original author(s) and the source are credited.

\section{References}

[1] Almeida KC, Salgado R (2000) Optimal power flow solutions under variable load conditions. IEEE Trans Power Syst 15(4):1204-1211

[2] Wood AJ, Wollenberg BF (2012) Power generation, operation, and control, 3rd edn. Wiley, New York

[3] Boyd S, Vandenberghe L (2009) Convex optimization. Cambridge University Press, New York

[4] Lima FGM, Galiana FD, Kockar I et al (2003) Phase shifter placement in large-scale systems via mixed integer linear programming. IEEE Trans Power Syst 18(3):1029-1034

[5] Holland JH (1975) Adaptation in natural and artificial systems: an introductory analysis with applications to biology, control, and artificial intelligence. University of Michigan Press, Ann Arbor
[6] Goldberg DE (1989) Genetic algorithms in search, optimization and machine learning. Addison-Wesley Longman Publishing Co, Reading

[7] Kennedy J, Eberhart R (1995) Particle swarm optimization. In: Proceedings of the IEEE international conference on neural networks, vol 4. Perth, WA, USA, 27 Nov-1 Dec 1995, pp 1942-1948

[8] Eberhart R, Kennedy J (1995) A new optimizer using particle swarm theory. In: Proceedings of the 6th international symposium on micro machine and human science (MHS '95), Nagoya, Japan, 4-6 Oct 1995, pp 39-43

[9] Storn R, Price K (1995) Differential evolution: a simple and efficient adaptive scheme for global optimization over continuous spaces. TR-95-012, International Computer Science Institut (ICSI), Berkeley, CA, USA

[10] Lakshminarasimman L, Subramanian S (2006) Short-term scheduling of hydrothermal power system with cascaded reservoirs by using modified differential evolution. IEE P-Gener Transm Distrib 153(6):693-700

[11] Wang Z, Chung CY, Wong KP et al (2008) Robust power system stabiliser design under multi-operating conditions using differential evolution. IET Gener Transm Distrib 2(5):690-700

[12] Vakula VS, Sudha KR (2012) Design of differential evolution algorithm-based robust fuzzy logic power system stabiliser using minimum rule base. IET Gener Transm Distrib 6(2):121-132

[13] Fogel LJ, Burgin GH (1969) Competitive goal-seeking through evolutionary programming. Air Force Cambridge Research Laboratories, Cambridge

[14] Back T, Fogel DB, Michalewicz Z (1997) Handbook of evolutionary computation. IOP Publishing Ltd, Bristol

[15] Dorigo M, Birattari M (2010) Ant colony optimization. In: Sammut C (ed) Encyclopedia of machine learning. Springer Science, Boston, pp p36-p39

[16] Hagan MT, Demuth HB, Beale MH (1996) Neural network design, vol 1. PWS Publishing Company, Boston

[17] Van Laarhoven PJM, Aarts EHL (1987) Simulated annealing: theory and applications. Reidel, Publishing Co, Dordrecht

[18] Glover F, Laguna M (1999) Tabu search. Kluwer Academic Publishers, Boston

[19] Han KH, Kim JH (2002) Quantum-inspired evolutionary algorithm for a class of combinatorial optimization. IEEE Trans Evolut Comput 6(6):580-593

[20] Han KH, Kim JH (2004) Quantum-inspired evolutionary algorithms with a new termination criterion, $H_{\varepsilon}$, gate, and two-phase scheme. IEEE Trans Evolut Comput 8(2):156-169

[21] Karaboga D, Basturk B (2008) On the performance of artificial bee colony (ABC) algorithm. Appl Soft Comput 8(1):687-697

[22] Iba K (1994) Reactive power optimization by genetic algorithm. IEEE Trans Power Syst 9(2):685-692

[23] Lai LL, Ma JT, Yokoyama R et al (1997) Improved genetic algorithms for optimal power flow under both normal and contingent operation states. Int $\mathrm{J}$ Electr Power Energy Syst 19(5):287-292

[24] Numnonda T, Annakkage UD (1999) Optimal power dispatch in multinode electricity market using genetic algorithm. Electr Power Syst Res 49(3):211-220

[25] Subbaraj P, Rajnarayanan PN (2009) Optimal reactive power dispatch using self-adaptive real coded genetic algorithm. Electr Power Syst Res 79(2):374-381

[26] Sailaja Kumari M, Maheswarapu S (2010) Enhanced genetic algorithm based computation technique for multi-objective optimal power flow solution. Int J Electr Power Energy Syst 32(6):736-742

[27] Wu QH, Cao YJ, Wen JY (1998) Optimal reactive power dispatch using an adaptive genetic algorithm. Int J Electr Power Energy Syst 20(8):563-569 
[28] Paranjothi SR, Anburaja K (2002) Optimal power flow using refined genetic algorithm. Electr Power Compon Syst 30(10):1055-1063

[29] Devaraj D, Yegnanarayana B (2005) Genetic-algorithm-based optimal power flow for security enhancement. IEE P-Gener Transm Distrib 152(6):899-905

[30] Bakirtzis AG, Biskas PN, Zoumas CE et al (2002) Optimal power flow by enhanced genetic algorithm. IEEE Trans Power Syst 17(2):229-236

[31] Gaing ZL, Chang RF (2006) Security-constrained optimal power flow by mixed-integer genetic algorithm with arithmetic operators. In: Proceedings of the Power Engineering Society general meeting. Montreal, Canada, 18-22 Jun 2006, 8 pp

[32] Yan W, Liu F, Chung CY et al (2006) A hybrid genetic algorithm-interior point method for optimal reactive power flow. IEEE Trans Power Syst 21(3):1163-1169

[33] Wankhade CM, Vaidya AP (2014) Optimal power flow using genetic algorithm: parametric studies for selection of control and states variables. Br J Appl Sci Technol 4(2):279-301

[34] Mahdad B, Srairi K, Bouktir T (2010) Optimal power flow for large-scale power system with shunt FACTS using efficient parallel GA. Int J Electr Power Energy Syst 32(5):507-517

[35] Zhao B, Guo CX, Cao YJ (2005) A multiagent-based particle swarm optimization approach for optimal reactive power dispatch. IEEE Trans Power Syst 20(2):1070-1078

[36] Hazra J, Sinha AK (2011) A multi-objective optimal power flow using particle swarm optimization. Eur Trans Electr Power 21(1):1028-1045

[37] Liang RH, Tsai SR, Chen YT et al (2011) Optimal power flow by a fuzzy based hybrid particle swarm optimization approach. Electr Power Syst Res 81(7):1466-1474

[38] Mahadevan K, Kannan PS (2010) Comprehensive learning particle swarm optimization for reactive power dispatch. Appl Soft Comput 10(2):641-652

[39] Abido MA (2011) Multiobjective particle swarm optimization for optimal power flow problem. In: Panigrahi B, Shi Y, Lim $\mathrm{MH}$ et al (eds) Handbook of swarm intelligence, vol 8. Springer, Berlin, pp p241-p268

[40] AlRashidi MR, El-Hawary ME (2007) Hybrid particle swarm optimization approach for solving the discrete OPF problem considering the valve loading effects. IEEE Trans Power Syst 22(4):2030-2038

[41] Mo N, Zou ZY, Chan KW et al (2007) Transient stability constrained optimal power flow using particle swarm optimisation. IET Gener Transm Distrib 1(3):476-483

[42] Onate Yumbla PE, Ramirez JM, Coello Coello CA (2008) Optimal power flow subject to security constraints solved with a particle swarm optimizer. IEEE Trans Power Syst 23(1):33-40

[43] Kang Q, Zhou MC, An J et al (2013) Swarm intelligence approaches to optimal power flow problem with distributed generator failures in power networks. IEEE Trans Automat Sci Eng 10(2):343-353

[44] Sivasubramani S, Swarup KS (2012) Multiagent based differential evolution approach to optimal power flow. Appl Soft Comput 12(2):735-740

[45] Amjady N, Sharifzadeh H (2011) Security constrained optimal power flow considering detailed generator model by a new robust differential evolution algorithm. Electr Power Syst Res 81(2):740-749

[46] Varadarajan M, Swarup KS (2008) Solving multi-objective optimal power flow using differential evolution. IET Gener Transm Distrib 2(5):720-730

[47] Abou El Ela AA, Abido MA, Spea SR (2010) Optimal power flow using differential evolution algorithm. Electr Power Syst Res 80(7):878-885
[48] Sayah S, Zehar K (2008) Modified differential evolution algorithm for optimal power flow with non-smooth cost functions. Energy Convers Manage 49(11):3036-3042

[49] Liang CH, Chung CY, Wong KP et al (2007) Study of differential evolution for optimal reactive power flow. IET Gener Transm Distrib 1(2):253-260

[50] Liang CH, Chung CY, Wong KP et al (2007) Parallel optimal reactive power flow based on cooperative co-evolutionary differential evolution and power system decomposition. IEEE Trans Power Syst 22(1):249-257

[51] Basu M (2008) Optimal power flow with FACTS devices using differential evolution. Int $\mathrm{J}$ Electr Power Energy Syst 30(2):150-156

[52] Cai HR, Chung CY, Wong KP (2008) Application of differential evolution algorithm for transient stability constrained optimal power flow. IEEE Trans Power Syst 23(2):719-728

[53] Sivasubramani S, Swarup KS (2011) Sequential quadratic programming based differential evolution algorithm for optimal power flow problem. IET Gener Transm Distrib 5(11): 1149-1154

[54] Yuryevich J, Wong KP (1999) Evolutionary programming based optimal power flow algorithm. IEEE Trans Power Syst 14(4):1245-1250

[55] Wong KP, Yuryevich J (1999) Optimal power flow method using evolutionary programming. In: Simulated evolution and learning, Proceedings of the 2nd Asia-Pacific conference on simulated evolution and learning (SEAL'98), LACS 1585, Canberra, Australia, 24-27 Nov 1998, pp 405-412

[56] Yan W, Lu S, Yu DC (2004) A novel optimal reactive power dispatch method based on an improved hybrid evolutionary programming technique. IEEE Trans Power Syst 19(2):913-918

[57] Ongsakul W, Tantimaporn T (2006) Optimal power flow by improved evolutionary programming. Electr Power Compon Syst 34(1):79-95

[58] Somasundaram P, Kuppusamy K, Kumudini Devi RP (2004) Evolutionary programming based security constrained optimal power flow. Electr Power Syst Res 72(2):137-145

[59] Sood YR (2007) Evolutionary programming based optimal power flow and its validation for deregulated power system analysis. Int J Electr Power Energy Syst 29(1):65-75

[60] Gnanadass R, Venkatesh P, Padhy NP (2004) Evolutionary programming based optimal power flow for units with nonsmooth fuel cost functions. Electr Power Compon Syst 33(3):349-361

[61] Gutierrez-Martinez VJ, Cañizares CA, Fuerte-Esquivel CR et al (2011) Neural-network security-boundary constrained optimal power flow. IEEE Trans Power Syst 26(1):63-72

[62] Venkatesh B (2003) Online ANN memory model-based method for unified $\mathrm{OPF}$ and voltage stability margin maximization. Electr Power Compon Syst 31(5):453-465

[63] Abbasy A, Hosseini SH (2007) Ant colony optimization-based approach to optimal reactive power dispatch: a comparison of various ant systems. In: Proceedings of the Power Engineering Society conference and exposition in Africa (PowerAfrica'07), Johannesburg, South Africa, 16-20 Jul 2007, 8 pp

[64] Vlachogiannis JG, Hatziargyriou ND, Lee KY (2005) Ant colony system-based algorithm for constrained load flow problem. IEEE Trans Power Syst 20(3):1241-1249

[65] Roa-Sepulveda CA, Pavez-Lazo BJ (2003) A solution to the optimal power flow using simulated annealing. Int $\mathrm{J}$ Electr Power Energy Syst 25(1):47-57

[66] Roy PK, Ghoshal SP, Thakur SS (2010) Biogeography based optimization for multi-constraint optimal power flow with emission and non-smooth cost function. Expert Syst Appl 37(12):8221-8228 
[67] Vlachogiannis JG, Lee KY (2008) Quantum-inspired evolutionary algorithm for real and reactive power dispatch. IEEE Trans Power Syst 23(4):1627-1636

[68] Ayan K, Kılıç U (2012) Artificial bee colony algorithm solution for optimal reactive power flow. Appl Soft Comput 12(5):1477-1482

[69] Ongsakul W, Bhasaputra P (2002) Optimal power flow with FACTS devices by hybrid TS/SA approach. Int J Electr Power Energy Syst 24(10):851-857

[70] Kumar S, Chaturvedi DK (2013) Optimal power flow solution using fuzzy evolutionary and swarm optimization. Int J Electr Power Energy Syst 47:416-423

[71] Chung CY, Liang CH, Wong KP et al (2010) Hybrid algorithm of differential evolution and evolutionary programming for optimal reactive power flow. IET Gener Transm Distrib 4(1):84-93

[72] Li Y, Wang Y, Li B (2013) A hybrid artificial bee colony assisted differential evolution algorithm for optimal reactive power flow. Int J Electr Power Energy Syst 52:25-33

[73] Vaisakh K, Srinivas LR (2011) Evolving ant direction differential evolution for OPF with non-smooth cost functions. Eng Appl Artif Intell 24(3):426-436

[74] Wolpert DH, Macready WG (1997) No free lunch theorems for optimization. IEEE Trans Evolut Comput 1(1):67-82

Ming NIU received the B.Eng. degree from Dalian University of technology, Dalian, China, in 2010, the MSc degree from The Hong Kong Polytechnic University, Hong Kong, in 2012. He is currently working toward the Ph.D. degree from The Hong Kong Polytechnic University. His research interests include power system planning and operation, computational intelligence, and optimization techniques applied in system networks.

Can WAN received the B.Eng. degree from Zhejiang University, Hangzhou, China, in 2008. He is currently pursuing his Ph.D. degree from The Hong Kong Polytechnic University. He was previously a Visiting Ph.D. with Centre for Electric Power and Energy, Technical University of Denmark, and State Key Laboratory of Power Systems, Department of Electrical Engineering, Tsinghua University. His research interests include power system uncertainty analysis and operation, grid integration of renewable energies, smart grids, machine learning and system engineering and control.

Zhao XU received the B.Eng. degree from Zhejiang University, Hangzhou, China, in 1996, the M.Eng. degree from National University of Singapore, Singapore, in 2002, and the Ph.D. degree from The University of Queensland, Australia, in 2006. He is now with The Hong Kong Polytechnic University. He was previously an Associate Professor with Centre for Electric Power and Energy, Technical University of Denmark. His research interests include demand side, grid integration of renewable energies and EVs, electricity market planning and management, and AI applications in power engineering. He is an editor of Electric Power Components and Systems. 